\title{
The distribution and effects of child mortality risk factors in Ethiopia: A comparison of estimates from DSS and DHS
}

\author{
Edward Fottrell ${ }^{1}$, Fikre Enquselassie ${ }^{2}$, Peter Byass ${ }^{1}$
}

\begin{abstract}
Objectives: To conduct a comparative analysis of the distribution and effects of under-five mortality correlates using Demographic and Health Survey (DHS) and Demographic Surveillance System (DSS) data from Ethiopia, and to investigate the methodological bias in DHS-based childhood mortality rates due to the impossibility of including children whose mothers were deceased.

Methods: Using all-cause under-5 mortality as an outcome variable, the distribution and effects of risk factors were modeled using survival analysis. All live births in rural Ethiopia in the 5-year period before the 2005 DSS+ survey and between 01/01/2000 and 31/12/2004 in the DSS in the Butajira Rural Health Program (in the Southern Nations, Nationalities, and People's (SNNP) region of Ethiopia) were included.

Results: Overall, similar estimates of hazard rate ratios were derived from both DHS and DSS data and the child mortality risk profile is similar between each data source, with multiple births and living in less populous households being significant risk factors for under-five mortality. Nevertheless, some notable differences were observed. The DSS data was more sensitive to local variations in population composition and health status, whilst the more dispersed DHS approach tended to average out local variation across the country. Excluding children whose mothers were deceased from the DSS analysis had no important effect on risk profiles or estimates of survival functions at age 5 years. DHS survival functions were somewhat lower than DSS estimates (BRHP $=0.87$, DHS rural Ethiopia $=0.67$, DHS $\mathrm{SNNP}=0.66$ ).

Conclusion: Despite differing methodologies, cross-sectional DHS and longitudinal DSS data produce estimates of the distribution and effects of under-five mortality risk factors that are broadly similar. The differing methodological characteristics of DHS and DSS mean that when combined, these two data sources have the potential to provide a comprehensive picture of national population composition and health status as well as the extent of local variation both of which are important for health monitoring and planning. [Ethiop. J. Health Dev. 2009;23(2):163-168]
\end{abstract}

\section{Introduction}

Methods to enhance knowledge of key health and demographic events in settings where widespread vital event and health surveillance systems are weak or nonexistent are key to understanding population health dynamics and associations between risk-factors and morbidity and mortality. Two such methods are Demographic Surveillance Systems (DSSs) and Demographic and Health Surveys (DHSs). Both DSS and DHS aim and produce representative data that may be used for health monitoring and planning, however the methodologies employed by these two approaches differ markedly.

Notwithstanding local and contextual variations, DSSs maintain regular surveillance of births, deaths and migrations and, frequently, social and economic correlates of population and health dynamics, in an open cohort population within a clearly circumscribed geographic area (1). This is achieved through an initial census followed by periodic household surveys. A major strength of DSSs is their unique suitability for acting as platforms for intervention studies in resource- and datapoor settings, and the robustness and representativeness of large DSS datasets have been documented $(2,3)$. Nevertheless, the fact that DSSs are localized systems and cover only a small proportion of total national populations has burdened DSSs with the task of demonstrating their usefulness in understanding population health on a wider scale and justifying the considerable costs and management that they require. In practice this has relied on comparisons with proxy goldstandards for population health profiles, most frequently DHSs (4-7).

DHSs are large, complex cross-sectional surveys that measure demographic and health parameters on a nationally representative sample (8). Nationally distributed cluster samples of households are performed at approximately five-year intervals, with each round drawing a new cross-section sample. The standard DHS survey consists of a household questionnaire and a women's questionnaire administered to a nationally representative sample of women aged 15-49 years. The women's questionnaire is used to gather information on complete birth histories to estimate infant and child mortality probabilities. The data produced from DHSs are generally accepted to be of high quality and representative at a national level, hence their use in assessments of DSS data.

In 2006, Hammer et al compared DHS and DSS data in Burkina Faso with respect to the effects of risk factors on childhood mortality (5). The study found that, despite the different nature of the cross-sectional and longitudinal data, similar results were obtained from each

${ }^{1}$ Umeå Centre for Global Health Research, Department of Public Health and Clinical Medicine, Umeå University, 90185, E-mail edward.fottrell@epiph.umu.se, Umeå, Sweden; ${ }^{2}$ School of Public Health, Addis Ababa University 
method. Inspired by the methods employed by Hammer et al (5), and following their recommendations that similar comparisons be conducted in other settings, this paper uses data from Butajira Rural Health Program (BRHP) DSS and the 2005 Ethiopian DHS+ survey to compare the distribution and effects of child mortality risk factors and to investigate the potential biases of DHS and DSS methods in estimating distributions of child mortality correlates and child survival. By comparing health measurements from different sources in this way we seek to assess the public health utility of available health and demographic data in Ethiopia while developing greater understanding of possible strengths and weaknesses of two different methods of measuring health in otherwise data-poor settings.

\section{Methods}

The first data source used in this study is the Butajira Rural Health Program (BRHP) in Ethiopia. BRHP is a DSS situated $130 \mathrm{~km}$ from Addis Ababa in the Southern Nations, Nationalities and Peoples (SNNP) region. Data have being collected through continuous communitybased surveillance of an open cohort population sample covering approximately $10 \%$ of the district. Household surveys have been conducted since 1987 and, to date, over 700,000 person-years of data have been collected. A primary concern of BRHP field operations is accurate linkages between individuals whilst rigorous quality control procedures and supervision ensure that the data are of a relatively high quality. The current resident population in the surveillance program is approaching 60,000 individuals. Butajira district was purposefully chosen as the location of the DSS as it was considered to be potentially representative of the wider Ethiopian population, including a mixture of religious and ethnic groups and a combination of highland and lowland as well as rural and semi-urban areas. Detailed descriptions of BRHP and its methods are available elsewhere (9-11).

The second data source is the 2005 DHS + cross-sectional survey of 13,721 households, 10,055 of which were classified as rural. Observations from Addis Ababa were excluded from the analysis as the capital city is markedly different in terms of health and risk factor exposure compared to the rest of the country. Data on household size, age of the women and their birth histories were gathered from a total of 14,070 eligible women aged 1549 years. SNNP region contributed a total of 2995 women to this total, which by design of the survey is intended to be representative of the province in which BRHP is situated (12).

As in Hammer et al's study (5), close comparability of the two datasets with regard to calendar period and setting was achieved by selecting all rural births for the five year period before the DHS interview, which was conducted between April and August 2005, and all births between $1^{\text {st }}$ January 2000 to $31^{\text {st }}$ December 2004 for the BRHP DSS data. Whilst the periods included do not match exactly, the large overlap enables a reasonable comparison of mortality and risk factors for the purposes of this study.

Childhood mortality risk factors of gender, age of mother, mother's education, multiple births, household size, mother's religion and mother's marital status were compared between the two data sources. Information on date of birth, date of last observation and vital status was also required for the analysis and was extracted from each database. Descriptive statistical methods were used to compare the distribution of the analysis variables in both data sets, comparing DSS with all rural DHS data, as well as with DHS data from the SNNP region alone.

Comparing the effect of major risk factors for childhood mortality was achieved by modeling mortality levels jointly for all strata of risk factor level using Cox proportional hazards regression and all-cause under-five mortality as the outcome variable following the methodology of Hammer et al (5). All risk factors were included in a single model as categorical variables. Observations were censored at death or the date of outmigration from the BRHP surveillance area, whichever occurred first.

The data were then used to estimate the methodological bias in DHS-based childhood mortality rates due to the impossibility of including children whose mothers were deceased at the time of the survey. Firstly, this was done by introducing the risk factor of 'mother has deceased' into the multivariate analysis using the DSS data. Secondly, five-year survival functions were estimated based on the DSS data with and without including individuals whose mothers were known to have died by the end of December 2004. Finally, all children who had no link to a mother in the DSS data set were assumed to have deceased or absent mothers and survival functions were estimated with these children excluded. By design, this sub-group could not have been included in the DHS survey, which relies on adult women's reports of their own reproductive history to measure child health. Whilst missing linkages to a mother in DSS data could be due to a number of reasons, given the long-term nature and frequent reconciliation of BRHP data, as well as strict quality control procedures, the assumption that an absent maternal linkage reflects absent or deceased mothers who would not have been included under DHS procedures is justified for the purposes of empirically investigating the influence of an absent mother on child survival.

\section{Results}

The selected DSS cohort consists of 6801 children born in the period $01 / 01 / 2000$ to $31 / 12 / 2004$. The data set consisted of 92 children (1.35\%) whose mothers had died by the end of December 2004 and 411 children (6.04\%) whose information was not linked to a mother. From the DHS dataset, information regarding 9821 children born by 6562 women within the five year period preceding the

Ethiop. J. Health Dev. 2009;23(2) 
DHS survey was used. The mean (and total) follow-up time was 2.21 years $(15,016$ person-years) for the BRHP data and 2.62 years $(25,709$ person-years) for the rural Ethiopian DHS data.

The distribution of parameters included in the analyses is shown in Table 1. There is generally good agreement in the overall distribution of parameters between the DHS data and BRHP data in most variables. There are however, notable exceptions. The proportion of multiple births in the BRHP data is approximately four times greater than in the estimates based on the DHS data. Also, according to the BRHP data, the majority of women live in households of between 1 to 5 individuals, unlike estimates based on the DHS data, which shows that the majority of women live in households of between 6 to 10 individuals. The biggest difference in distributions between data sources is in terms of religion. Islam is the dominant religion among women in BRHP, with almost $70 \%$ of women being recorded as Muslim. In contrast, there is a fairly even split between Orthodox Christianity and Islam as the dominant religion according to the rural DHS data, and Orthodox Christianity is the dominant religion according to the SNNP DHS data, with approximately $60 \%$ of women reporting this as their religion. Proportions of unmarried and educated women also appear to be over- and underestimated, respectively, in the BRHP data compared to DHS data.

Table 1: Distributions from rural Ethiopian DHS data, SNNP DHS data and BRHP DSS data.

\begin{tabular}{|c|c|c|c|c|c|c|}
\hline \multirow[b]{2}{*}{ Characteristics } & \multicolumn{2}{|c|}{ DHS+Rural Ethiopia } & \multicolumn{2}{|c|}{ DHS+SNNP } & \multicolumn{2}{|c|}{ BRHP } \\
\hline & $\begin{array}{l}\text { Total No. of } \\
\text { Children (\%) }\end{array}$ & $\begin{array}{l}\text { No. of } \\
\text { Deaths }\end{array}$ & $\begin{array}{l}\text { Total No. of } \\
\text { Children (\%) }\end{array}$ & $\begin{array}{l}\text { No. of } \\
\text { Deaths }\end{array}$ & $\begin{array}{l}\text { Total No. of } \\
\text { Children (\%) }\end{array}$ & $\begin{array}{l}\text { No. of } \\
\text { Deaths }\end{array}$ \\
\hline \multicolumn{7}{|l|}{ Gender } \\
\hline Male & $5007(51.0)$ & 487 & $841(48.6)$ & 84 & $3474(51.1)$ & 344 \\
\hline Female & $4814(49.0)$ & 369 & $889(51.4)$ & 78 & 3327 (48.9) & 351 \\
\hline \multicolumn{7}{|l|}{ Year of Birth } \\
\hline 2000 & $209(2.1)$ & 26 & $34(2.0)$ & 2 & $1404(20.6)$ & 180 \\
\hline 2001 & 2109 (21.5) & 233 & $365(21.1)$ & 46 & $1501(22.1)$ & 172 \\
\hline 2002 & $1962(20.0)$ & 169 & $357(20.6)$ & 34 & $1314(19.3)$ & 126 \\
\hline 2003 & $1864(19.0)$ & 159 & $287(16.6)$ & 33 & $1179(17.3)$ & 126 \\
\hline 2004 & $1890(19.2)$ & 169 & $323(18.7)$ & 31 & $1403(20.6)$ & 91 \\
\hline 2005 & $1787(18.2)$ & 100 & $364(21.0)$ & 16 & - & - \\
\hline \multicolumn{7}{|c|}{ Age of Mother at Birth (years) } \\
\hline$<18$ & $739(7.5)$ & 95 & $286(16.5)$ & 36 & $223(3.3)$ & 17 \\
\hline $18-35$ & $7965(81.1)$ & 653 & $701(40.5)$ & 54 & $4447(65.4)$ & 435 \\
\hline$\geq 36$ & $1117(11.4)$ & 108 & $743(43.0)$ & 72 & $1252(18.4)$ & 143 \\
\hline Unknown & 0 & - & 0 & - & $879(12.9)$ & 100 \\
\hline \multicolumn{7}{|l|}{ Multiple Birth } \\
\hline No & $9645(98.2)$ & 811 & $1669(98.0)$ & 154 & $5942(87.4)$ & 567 \\
\hline Yes & $176(1.8)$ & 45 & $34(2.0)$ & 8 & $448(6.6)$ & 92 \\
\hline Unknown & 0 & - & 0 & - & $411(6.0)$ & 36 \\
\hline \multicolumn{7}{|c|}{ No. of Household Members } \\
\hline $1-5$ & $4313(43.9)$ & 494 & $721(41.7)$ & 84 & $3217(47.3)$ & 357 \\
\hline $6-10$ & $5201(53.0)$ & 347 & $936(54.1)$ & 71 & $3115(45.8)$ & 262 \\
\hline$>11$ & $307(3.1)$ & 15 & $73(4.2)$ & 7 & $469(6.9)$ & 76 \\
\hline \multicolumn{7}{|l|}{ Mother's Religion } \\
\hline Orthodox & $3858(39.3)$ & 341 & $364(21.0)$ & 38 & $1119(16.5)$ & 123 \\
\hline Other Christian & $1868(19.0)$ & 158 & $1034(59.8)$ & 92 & $215(3.2)$ & 15 \\
\hline Muslim & $3846(39.2)$ & 341 & $232(13.4)$ & 22 & $4744(69.8)$ & 484 \\
\hline Other & $245(2.5)$ & 16 & $100(5.8)$ & 10 & $6(0.1)$ & 1 \\
\hline Unknown & 0 & - & 0 & - & $717(10.5)$ & 72 \\
\hline \multicolumn{7}{|l|}{ Mother's Marital Status } \\
\hline Married & $9185(93.5)$ & 786 & 1665 (96.2) & 152 & $5266(77.4)$ & 528 \\
\hline Not married & $636(6.5)$ & 70 & $65(3.8)$ & 10 & $774(11.4)$ & 88 \\
\hline Unknown & - & - & 0 & - & $761(11.2)$ & 79 \\
\hline \multicolumn{7}{|l|}{ Mother's Education Level } \\
\hline No formal education & $7584(77.2)$ & 712 & $1291(74.6)$ & 134 & $4334(63.7)$ & 440 \\
\hline Primary & $1539(15.7)$ & 111 & $364(21.0)$ & 25 & $203(3.0)$ & 11 \\
\hline Secondary & $698(7.1)$ & 33 & 75 (4.3) & 3 & $37(0.5)$ & 1 \\
\hline Unknown & 0 & - & 0 & - & 2227 (32.8) & 243 \\
\hline \multicolumn{7}{|l|}{ Mother Known } \\
\hline Yes & $9821(100)$ & 856 & $1730(100)$ & 162 & $6390(94.0)$ & 659 \\
\hline No & 0 & - & 0 & - & $411(6.0)$ & 36 \\
\hline \multicolumn{7}{|l|}{ Mother Alive } \\
\hline Yes & $9821(100)$ & 856 & $1730(100)$ & 162 & $6298(92.6)$ & 621 \\
\hline No & 0 & - & 0 & - & $92(1.4)$ & 38 \\
\hline Unknown & 0 & - & 0 & - & $411(6.0)$ & 36 \\
\hline Total & $9821(100)$ & 856 & $1730(100)$ & 162 & $6801(100)$ & 695 \\
\hline
\end{tabular}


Table 2 shows hazard rate ratios adjusted for all other variables shown. The child mortality risk profile is similar between each data source, with multiple births and living in less populous households being significant risk factors for under-five mortality. Nevertheless, some differences can be observed between data sources. For example, sex of the child is a significant risk factor according to the rural DHS data, however this risk is not apparent in the more local SNNP DHS or BRHP DSS data. The risk conferred by older mothers is significant in both the rural DHS and BRHP data, but is not identified when only the SNNP DHS dataset is used, perhaps because of the relatively small sample size and the more even distribution of women into the different age categories in this sample.

The results of a regression model for the BRHP data including death of the mother as a risk factor indicate that a mother's survival has a powerful effect on her child's survival $\quad(H R=5.35,95 \% \quad C I: 3.63-7.87, \quad \mathrm{p}<0.001)$.
Excluding the 92 children whose mothers were deceased by the end of 2004 from the analysis had no important effect on the risk profiles derived in the Cox regression analysis. Having no linkage to a mother in the BRHP dataset has no significant association with child mortality $(\mathrm{HR}=1.15,95 \% \mathrm{CI}: 0.82-1 / 61, \mathrm{p}=0.411)$ and excluding these children has no important influence on the hazard ratios and confidence intervals of factors shown in Table 2.

The estimates of survival functions at age 5 years were negligibly higher in children whose mothers were still living at the end of December 2004 compared to all children ( 87 vs. 0.88 ), thereby indicating a very small underestimation in the DHS data caused by not including children of deceased mothers. Overall, however, DHS survival functions were somewhat lower than DSS estimates $(\mathrm{BRHP}=0.87$, DHS rural Ethiopia $=0.67$, DHS $\mathrm{SNNP}=0.66)$.

Table 2: Rate ratio estimates based on survival analysis for rural Ethiopian DHS data, SNNP DHS data and BRHP DSS data

\begin{tabular}{|c|c|c|c|c|c|c|}
\hline \multirow[b]{2}{*}{ Characteristics } & \multicolumn{2}{|c|}{ DHS+Rural Ethiopia } & \multicolumn{2}{|c|}{ DHS+SNNP } & \multicolumn{2}{|c|}{ BRHP } \\
\hline & $\begin{array}{l}\text { Hazard Ratio } \\
(95 \% \mathrm{Cl})\end{array}$ & $p>\chi^{2}$ & $\begin{array}{l}\text { Hazard Ratio } \\
(95 \% \mathrm{Cl})\end{array}$ & $p>\chi^{2}$ & $\begin{array}{l}\text { Hazard Ratio } \\
(95 \% \mathrm{Cl})\end{array}$ & $p>\chi^{2}$ \\
\hline \multicolumn{7}{|l|}{ Gender } \\
\hline Male & $0.76(0.67-0.87)$ & $<0.001$ & $0.85(0.62-1.15)$ & 0.289 & $0.93(0.76-1.13)$ & 0.452 \\
\hline Female & 1.00 & & 1.00 & & 1.00 & \\
\hline \multicolumn{7}{|c|}{$\begin{array}{l}\text { Age of Mother at Birth } \\
\text { (years) }\end{array}$} \\
\hline$<18$ & $1.15(0.92-1.43)$ & 0.215 & $1.28(0.75-2.18)$ & 0.368 & $0.60(0.28-1.27)$ & 0.182 \\
\hline $18-35$ & 1.00 & & 1.00 & & 1.00 & \\
\hline$\geq 36$ & $1.46(1.18-1.80)$ & 0.001 & $1.28(0.79-2.10)$ & 0.319 & $1.27(1.01-1.60)$ & 0.038 \\
\hline \multicolumn{7}{|l|}{ Multiple Birth } \\
\hline No & 1.00 & & 1.00 & & 1.00 & \\
\hline Yes & $3.50(2.58-4.75)$ & $<0.001$ & $4.06(1.92-8.56)$ & $<0.001$ & 2.27 (1.74-2.97) & $<0.001$ \\
\hline \multicolumn{7}{|l|}{$\begin{array}{l}\text { No. of Household } \\
\text { Members }\end{array}$} \\
\hline $1-5$ & 1.00 & & 1.00 & & 1.00 & \\
\hline $6-10$ & $0.48(0.41-0.55)$ & $<0.001$ & $0.55(0.40-0.78)$ & 0.001 & $0.75(0.61-0.93)$ & 0.008 \\
\hline$>11$ & $0.34(0.20-0.57)$ & $<0.001$ & $0.67(0.30-1.48)$ & 0.316 & $1.17(0.81-1.70)$ & 0.411 \\
\hline \multicolumn{7}{|l|}{ Religion } \\
\hline Orthodox & $0.98(0.84-1.22)$ & 0.826 & $1.36(0.80-2.32)$ & 0.251 & $1.01(0.78-1.31)$ & 0.935 \\
\hline Other Christian & $1.01(0.83-1.22)$ & 0.953 & $1.02(0.64-1.64)$ & 0.921 & $0.85(0.46-1.55)$ & 0.593 \\
\hline Muslim & 1.00 & & 1.00 & & 1.00 & \\
\hline Other & $0.68(0.41-1.12)$ & 0.131 & $1.08(0.51-2.28)$ & 0.849 & $2.80(0.39-20.0)$ & 0.304 \\
\hline \multicolumn{7}{|c|}{ Mother's Marital Status } \\
\hline Married & 1.00 & & 1.00 & & 1.00 & \\
\hline Not married & $0.97(76-1.25)$ & 0.819 & $1.47(0.77-2.82)$ & 0.243 & $1.14(0.81-1.59)$ & 0.453 \\
\hline \multicolumn{7}{|c|}{$\begin{array}{l}\text { Mother's Education } \\
\text { Level }\end{array}$} \\
\hline No education & $1.97(1.39-2.81)$ & $<0.001$ & $2.18(0.69-6.91)$ & 0.186 & $3.03(0.42-21.7)$ & 0.269 \\
\hline Primary & $1.62(1.09-2.40)$ & 0.016 & $1.61(0.48-5.38)$ & 0.442 & $1.33(0.17-10.6)$ & 0.790 \\
\hline Secondary & 1.00 & & 1.00 & & 1.00 & \\
\hline
\end{tabular}




\section{Discussion}

By comparing the distribution of major child mortality risk factors and examining the effect of these factors on child survival, this study demonstrates ways in which cross-sectional and longitudinal population surveillance methods complement and contradict one another and how ultimately they can enhance understanding of health in resource-poor settings where routine health monitoring is absent. Applying Hammer et al's (5) methods to data from Ethiopia adds further insight into the biases of DHS and DSS methods in general and may also be of practical use to health planners in Ethiopia, and perhaps elsewhere, tasked with basing local and national health policy decisions on limited population health information.

DHS surveys are useful tools for assessing health at a national level. Whilst DSSs have more scope to detect the extent of local variations in population composition and health status, the more dispersed DHS approach averages out local variations across a region or nation. As illustrated by the differences in the distribution of religion (Table 1), Ethiopia is a vastly diverse country and national estimates can mask local variations and the population compositions of localized communities, even if such communities are themselves geographically and ethnically diverse, as is the case in Butajira. Therefore, general population distributions and uniformity of certain parameters within localities are important determinants of whether nationally derived estimates can be applied locally and vice versa. For example, relatively even distributions of sex composition mean that all methods are likely to represent the overall population well whereas religion, which may be less diverse locally than nationally, may be better represented by a more distributed sampling procedure as used in the DHS approach. A similar conclusion highlighting the importance of parameter distributions was drawn following an in depth investigation into the effects of different sampling approaches on the representativeness of DSS data (2).

The specific methodologies of DHS and DSS are associated with specific biases. DHS surveys rely on the willingness of mothers to cooperate and their ability to recall and accurately report their birth histories and the vital status of their child. The longitudinal, prospective nature of the BRHP data, usually gathered by the same fieldworker visiting every household every three months, guarantees high participation rates and also increases the likelihood that important life events, including pregnancies, will be observed even if not directly reported by the respondents. These differences may partly explain some of the differences in risk factor distributions observed in this study. For example, the greater proportion of twins in BRHP compared to the DHS data could partly be due to reporting bias in the DHS survey whereby there may be a tendency to only report healthy, live births, and since multiple births are at greater risk of dying, they may be underreported. In a DSS setting, pregnancies are more likely to be noticed and thus all birth outcomes are more likely to be recorded.

The snap-shot cross-sectional approach of DHS may also explain the different distributions in household size observed between DHS and BRHP. DHS relies on eligible adult females being present when the data are collected. Although DHS procedures allow up to three attempts to be made to contact any individual, it may be easier to complete interviews with adult females residing in larger households, whereby someone is more likely to be at home when a fieldworker calls and other household members are more likely to be on hand to enable the eligible women to participate in the survey - both of these factors may systematically increase the likelihood of including larger households in the final sample, unlike in DSS methods where data will ultimately be collected from every household in the surveillance area. The lower proportion of unmarried and uneducated women in the DHS samples may also be due to a combination of response and reporting biases which are diminished by the longitudinal, prospective nature of DSS methods.

Despite the above differences, and in accordance with the results obtained by Hammer et al in 2006 (5), survey results from both DHS and DSS are broadly comparable and general public health conclusions that would be drawn from the data would be unlikely to differ much between data source. Whilst it is possible that similarities between data sources are coincidental, given the purposeful initial selection of Butajira as a district that could be nationally representative, such coincidence is unlikely (4) and drawing comparisons between national and regional DHS data and localized DSS data is reasonable.

The differing yet complementary characteristics of DHS and DSS mean that, when combined, these two data sources have the potential to characterize national population composition and health status as well as the extent of local variation-both of which are important for health monitoring and planning. Distributed DSS methods, whereby DSS-nodes are more geographically dispersed, may improve the representatively of current DSS health and demographic estimates, thereby enhancing their utility; such approaches warrant serious consideration $(2,13)$. In the meantime, further investigation into the unique, practical ways in which the combination and comparison of existing datasets may be able to support health systems is needed so that surveillance in otherwise data-poor countries becomes a synthesis of all available and relevant data. Maximizing the utility of all data in this way will help to ensure that the task of surveillance does not end with simply measuring risk factors and counting deaths but with making these data and these deaths count in terms of improving population health.

Ethiop. J. Health Dev. 2009;23(2) 


\section{Acknowledgements}

This work was undertaken within the Umeå Centre for Global Health Research, with support from FAS, the Swedish Council for Working life and Social Research (grant no. 2006-1512).

\section{References}

1. Indepth. International Network of Field Sites With Continuous Demographic Evaluation of Populations and Their Health in Developing Countries. 2008 [cited $1^{\text {st }}$ September 2008]; Available from: www.indepth-network.org.

2. Fottrell E, Byass P. Population survey sampling methods in a rural African setting: measuring mortality. Population Health Metrics 2008;6(2).

3. Fottrell E, Byass P, Berhane Y. Demonstrating the robustness of population surveillance data: implications of error rates on demographic and mortality estimates. BMC Medical Research Methodology 2008;8(13).

4. Byass P, Worku A, Emmelin A, Berhane Y.DSS and DHS: longitudinal and cross-sectional viewpoints on child adolescent mortality in Ethiopia. Population Health Metrics 2007;5(12).

5. Hammer GP, Kouyaté B, Ramroth H, Becher H. Risk factors for childhood mortality in sub-Saharan Africa: a comparison of data from a Demographic and Health Survey and from a Demographic Surveillance System. Acta Tropica 2006;98:212-8.

6. Bairagi R, Becker S, Kantner A, Allen KB, Datta A, Purvis K. An evaluation of the 1993-4 Bangladesh Demographic and Health Survey within the Matlab area. Asia Pacific Population Research Abstracts. 1997; 11:1-2.
7. Nhacolo AQ, Nhalungo DA, Sacoor CN, Aponte JJ, Thompson R, Alonso P. Levels and trends of demographic indices in southern rural Mozambique: evidence from demographic surveillance in Manhiça district. BMC Public Health 2006;(291).

8. DHS. Measure DHS. 2008 [cited $1^{\text {st }}$ September 2008]; Available from: www.measuredhs.com.

9. Berhane Y, Byass P. Chapter 8: Butajira DSS, Ethiopia. Population and Health in Developing Countries: Volume 1. Ottawa: IDRC 2002.

10. Berhane Y, Wall S, Kebede D, Emmelin A, Enquselassie F, Byass P, et al. Establishing an epidemiological field laboratory in rural areaspotentials for public health research and interventions: The Butajira Rural Health Programme 1987-99. Ethiop. J. Health Dev. 1999;13:(Special Issue).

11. Byass P, Berhane Y, Emmelin A, Kebede D, Andersson $T$, Högberg $U$, et al. The role of demographic surveillance systems (DSS) in assessing the health of communities: an example from rural Ethiopia. Public Health 2002;116:145-50.

12. DHS. Ethiopia Demographic and Health Survey 2005. Addis Ababa, Ethiopia and Calverton, Maryland, USA: Central Statistics Agency and ORC Macro 2006.

13. Fottrell E. Dying to Count: Mortality Surveillance Methods in Resource-Poor Settings. Umeå: University of Umeå; 2008. Available from: http://ww.diva-portal.Org/umu/abstract.Xsq1?dbid= 1544. 